\title{
ROAD NETWORK IDENTIFICATION AND EXTRACTION IN SATELLITE IMAGERY USING OTSU'S METHOD AND CONNECTED COMPONENT ANALYSIS
}

\author{
P. Yadav ${ }^{1 *}$, S. Agrawal ${ }^{2}$ \\ ${ }^{1}$ GIS Cell, Motilal Nehru National Institute of Technology, Allahabad-211004, U.P., India - priti6400307@gmail.com \\ ${ }^{2}$ GIS Cell, Motilal Nehru National Institute of Technology, Allahabad-211004, U.P., India - sonam@mnnit.ac.in
}

Commission V, WG V/5

KEY WORDS: Road identification, road extraction, Otsu's method, connected component analysis, morphological operations.

\begin{abstract}
:
As the high resolution satellite images have become easily available, this has motivated researchers for searching advanced methods for object detection and extraction from satellite images. Roads are important curvilinear object as they are a used in urban planning, emergency response, route planning etc. Automatic road detection from satellite images has now become an important topic in photogrammetry with the advances in remote sensing technology. In this paper, a method for road detection and extraction of satellite images has been introduced. This method uses the concept of histogram equalization, Otsu's method of image segmentation, connected component analysis and morphological operations. The aim of this paper is to discover the potential of high resolution satellite images for detecting and extracting the road network in a robust manner.
\end{abstract}

\section{INTRODUCTION}

Satellite images obtain information of areas which are difficultto-reach, provide huge volume of data and monitor events and areas without any interference. The new sensors in satellites provide high resolution imagery with better quality. This has increased the potential for analysis tools to identify and extract linear features (Singh and Garg, 2013). For transportation and city planning, roads are very essential linear features. Automatic road detection from satellite images has now become an important topic in photogrammetry after remote sensing technology development. In this research paper, road detection and extraction from satellite images has been performed. In the methodology histogram equalization, Otsu's method of image segmentation, connected component analysis and morphological operations are used.

\section{THEORITICAL BACKGROUND}

Satellite images often contain noises. Therefore, these images are preprocessed and enhanced before the extraction of objects. In image enhancement, digital images are altered and its visual interpretability is improved. Therefore, resultant images are more appropriate for analysis. Contrast enhancement is one of image enhancement technique. It improves the appearance of object and the brightness between object and its backgrounds (Chalekar and Yengantiwar, 2014).

Satellite images often contain several objects. Visual detection of these objects by humans is a slow, expensive and endless routine job. Therefore automatic object detection with the help of computers was developed. Object detection algorithms localise objects and annotates them with an object class label. Both tasks face many difficulties. On one hand, the detection method must be robust for different images or varying illumination conditions and it must account for changes in the object. On the other hand, these objects may appear of any size and at anywhere in an image. Hence for practical systems, efficiency is very important factor (Kapoor et al., 2014). The objects in satellite images are divided into three main categories of point, line and area type (Mohammadzadeh et al., 2009). Linear features are of interest to a lot of researchers. Mathematically, linear features are described by arbitrary curves or straight lines. These features are used in GIS, digital mapping, monitoring the dynamic changes of water and for updating GIS vector data (Zhang et al., 2013). It can also used in transportation database, city planning, military field and map updating (Wang et al., 2016). Linear pattern recognition has many practical applications like road detection, river detection, railroads detection, plant root analysis, retinal vessel extraction etc.

Automatic road detection from satellite images has now become an important topic in photogrammetry after the advances in remote sensing technology. An automatic process of road extraction is needed by GIS for continuous data update (Hu et al., 2009). Methods for road extraction can be semi-automatic or fully automatic. Semi-automatic detection method requires human interaction while automatic detection method does not require human interaction (Lin et al., 2009).

In last three decades, a lot of research has been carried out in automatic methods for road detection which has resulted in many algorithms and strategies (Mena, 2003). Gruen and Li (1997) have given a method that involved dynamic programming. Keaton and Brokish (2002) suggested a method for road extraction in which a seed point is selected from the road and then a level set method is used to grow the region. Iterative smoothing is performed which further improved the extracted section and then road centreline is estimated accurately. He et al. (2004) developed a road area detection algorithm which has two modules. In first module, scene area was reduced for the computation of mean and variance of Gaussian distribution. In second module the road area was extracted and then boundaries that fit the result of road extraction were reinforced. Fuzzy method was applied for road extraction in a paper (Mohammadzadeh et al., 2009, 2007). 
Hu et al. (2009) has adopted Least Squares Template Matching (LSTM) for fitting the linear objects with a Scalable Slope Edge (SSE) model. A method was presented for linear feature extraction which has integrated the SSE with a LSTM model for processing noisy images and blurred edges. Li and Briggs (2009) gave an algorithm in which edge filtering was performed to extract roads. Stage segmentation, grouping and optimization were then applied for roads that are usually harder to identify because of noise and also to fill big gaps. Wu and ShuFeng (2009) have proposed an algorithm for unstructured road detection. In this method, median filter is applied on original images to suppress the stochastic type of noise and then multithreshold is selected by Otsu method on the basis of two-peak method. Finally, the primary edge is detected with the help of canny operator and operations of mathematics morphology are used, which results in clear road image.

Valero et al. (2010) have used mathematical morphology in road detection. Parvathi et al., (2010) have proposed a method which extracted linear anthropogenic objects in hilly areas. Object and ground separation was done by employing wavelets in hilly terrain. It extracted desired objects in which markercontrolled watershed segmentation is employed. Wang and Zhang (2011) have given the gradient-based model in which used extended Snakes model for positional optimization. Alvarez et al., (2009) combined a classifier determining road class-likelihood with an illumination invariant feature space to provide reliable results regardless of shadows and lighting variations. Wang et al. (2013) have used a knowledge-based approach for road detection. Singh and Garg (2013) applied morphological operations and adaptive global thresholding on images to extract roads. In another paper, they used a two-stage framework using fuzzy clustering, region part-segmentation, segment linking and medial axis transformation (Singh and Garg, 2014). Sujatha and Selvathi, (2015) have extracted road centerline automatically from satellite image. This method has three steps which are road region segmentation, connected component operations to extract the connected component of road from segmented part and then non road pixel removal by morphological operations. Several other papers also used connected component analysis and segmentation (Reddy and Padmaja, 2016; Wang et al., 2016).

J. Wang et al. (2016) proposed an object-based method in which extraction of spatial texture features was done by specific spatial statistics and then derived texture was added to spectral bands for road extraction. Maboudi et al. (2017) have developed object-based image analysis (OBIA) method in which object-based information is implanted in the ant colony optimization algorithm to handle the problem of extraction of road network.

In this way several techniques were developed or taken from other fields for road extraction. They used image processing along with mathematics, soft computing, artificial neural network etc. This paper also developed and implemented a method for road detection and extraction.

\section{METHODOLOGY}

In this paper, a novel method has been proposed for detecting and extracting the road network from high resolution satellite images. In this method, the input image is first preprocessed. After that a threshold is computed by Otsu's method $(\mathrm{Qu}$ and Zhang, 2010). Using this threshold, the gray image is converted

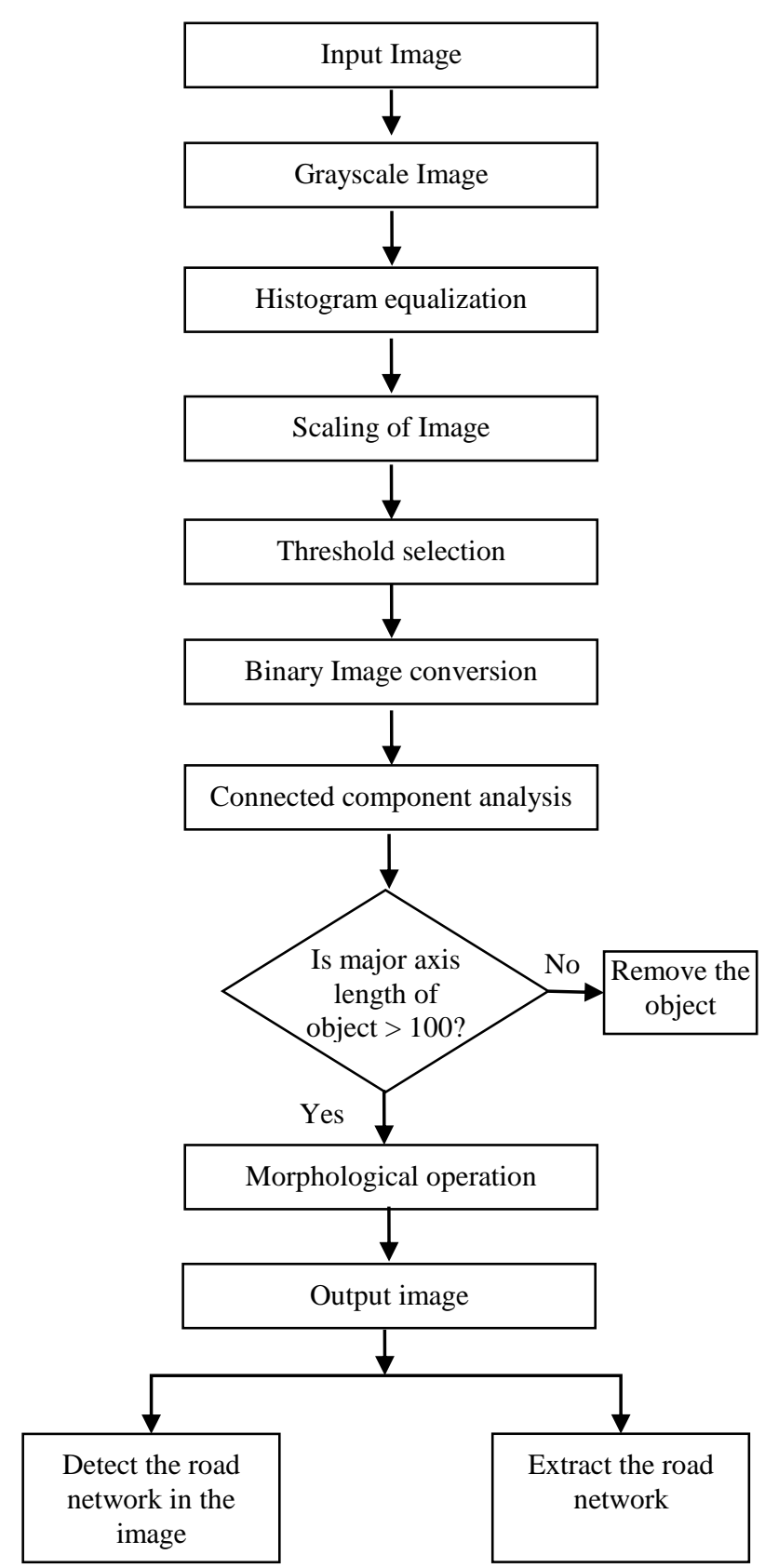

Figure 1: Road detection and extraction methodology

into binary image and it contains only black and white pixels. Connected component analysis is then performed. After that, the image is processed by morphological operators and thus a finer road network is extracted. This extracted network is then superimposed with the input image to highlight the road network in the input image. Figure 1 shows the overall methodology. Its steps are discussed in detail below:

\subsection{Image Preprocessing}

First of all, preprocessing is performed on the image. This step removes the noise from the image and enhances the contrast of the image. Preprocessing of the image has following steps:

\subsubsection{Grayscale conversion}

Colored images have three independent channels: red, green and blue. This increases the cost, processing time and 
complexity of the image. Grayscale images are single channel images on which contrast, shape, edges, etc can be studied without using color channels. Therefore the coloured images are converted into grayscale images.

\subsubsection{Histogram equalization}

Histogram equalization is a technique which remaps the input image pixels so that almost uniform histogram may be achieved. It helps in enhancing the contrast of the image. It is applied on the grayscale image.

\subsubsection{Image Scaling}

Scaling of image is performed to remove the problem of quantization and aliasing (Alemu, 2016). Quantization is a process in which values of a range of pixels are replaced by a specific value. Aliasing problem arises when various signals are sampled and it becomes impossible to differentiate these signals (Gioi et al., 2008).

\subsection{Binary image conversion}

\subsubsection{Selection of threshold}

Otsu's method of image segmentation is used for threshold selection. Otsu's method was given by N. Otsu in 1979. Otsu's algorithm selects threshold automatically. It is effective, fast, simple and stable. In Otsu's algorithm, a global threshold is computed which is later used to convert image into binary image. This global threshold is a positive scalar.

\subsubsection{Binarization}

After threshold selection, image is converted into another image where all pixel values which are greater than the threshold are replaced by 1 and rest pixel values are replaced by 0 . As a result of this step, a black and white image is formed.

\subsection{Connected component analysis}

Connected pixels are set of pixels which are not divided by boundary. Its basic idea is an identification logic whose role is to detect the components which are single, broken or connected characters (Vijayan et al., 2016). After finding connected components, trivial opening is performed. Trivial opening extracts the connected component based on some criteria. If connected component of image satisfies the criteria $\mathrm{T}$, then component is preserved, if not then component is removed. The criteria are selected so that only long features can be extracted. Hence, the criterion ( $\mathrm{T}$ ) selected for trivial opening in this work is major axis length. The longest diameter of an ellipse is called its major axis. If $\mathrm{P}$ is the image and $\mathrm{P}(\mathrm{i})$ is a connected component of the image, then the trivial operation is performed by following equation (Sujatha and Selvathi, 2015) which gives the connected component $\left(\mathrm{R}_{0}\right)$ :

$\mathrm{R}_{0}=\{\mathrm{P} \mid$ Long axis of minimum ellipse enclosing $\mathrm{P}(\mathrm{i}) \geq \mathrm{T}\}$

\subsection{Morphological operation}

After connected component analysis, the extracted roads still contain some holes and noises. This is so because connected component sometimes cannot identify small ground objects like building, lanes, vehicles, etc. To eliminate them and improve the accuracy of the results, the extracted results are processed by the various operations of mathematical morphology like opening, closing, dilation and erosion. Matheron and Serra introduced method of mathematical morphology by in 1964 . Basic idea of this method is the application of mathematical morphology for image processing and analysis. Morphology is an approach in which objects and object features are identified through their shape (Kaur and Ritika, 2013).

\subsubsection{Area opening}

This operation removes those connected components which have less number of pixels than a threshold (https://in.mathworks.com).

\subsubsection{Thinning operation}

This morphological operation reduces or thins the connected components to lines. Pixels are removed in such manner that an object which does not contain any holes reduces to a minimally connected stroke while object with holes reduces to a connected loop intermediate between holes and the external boundary.

\subsubsection{Opening operation}

This operation is a process of erosion followed by dilation. Same structuring element is used for both erosion and dilation.

\subsubsection{Closing operation}

This operation is a process of erosion followed by dilation. Same structuring element is used for both erosion and dilation.

\subsection{Detection of road network}

For detecting the road network in the original image, individual red, green and blue color channels are extracted from original RGB image. Road pixels are assigned red colours. This creates an image with red colored road network with white background. Then this red colored road network is superimposed with initial Grayscale image. The image is shown as a Grayscale image with its road network colored in red.

\subsection{Extraction of road network}

After morphological operation, the roads in single pixel are formed. To get the extracted road network, black color is assigned to all pixels of road network and white color is assigned to rest of the pixels. Therefore, an extracted road network is generated in which roads are shown in black pixels with white background.

\section{GRAPHICAL USER INTERFACE}

Graphical User Interface is created in MATLAB. It contains a figure window in which menus, text, graphics, buttons etc. are provided. To open GUI, select File/New/GUI from menu or type "guide" on Command Window and select "Create New GUI". Layout Editor is then opened in which the appearance of GUI can be designed. On the left of the Layout Editor some buttons are given for inserting objects like toggle buttons, push buttons, static text, axes box etc. GUI is then created with the help of Layout Editor. Property Inspector is used to alter the object properties like text font, color etc. For saving a GUI, click Save As from File menu and type the name of file without any extension (Hunt et al., 2012). After saving it, two types of files are created binary file with '.fig' extension and an M-file with '.m' extension. When the M-file is run from tools menu, GUI appears with selected buttons and menus of layout editor. To assign the functionality to the GUI buttons callback function is used. Commands are added to the buttons after opening the M-file. Now it is saved and executed. GUI window is then opened and on clicking the buttons desired operation are performed. 


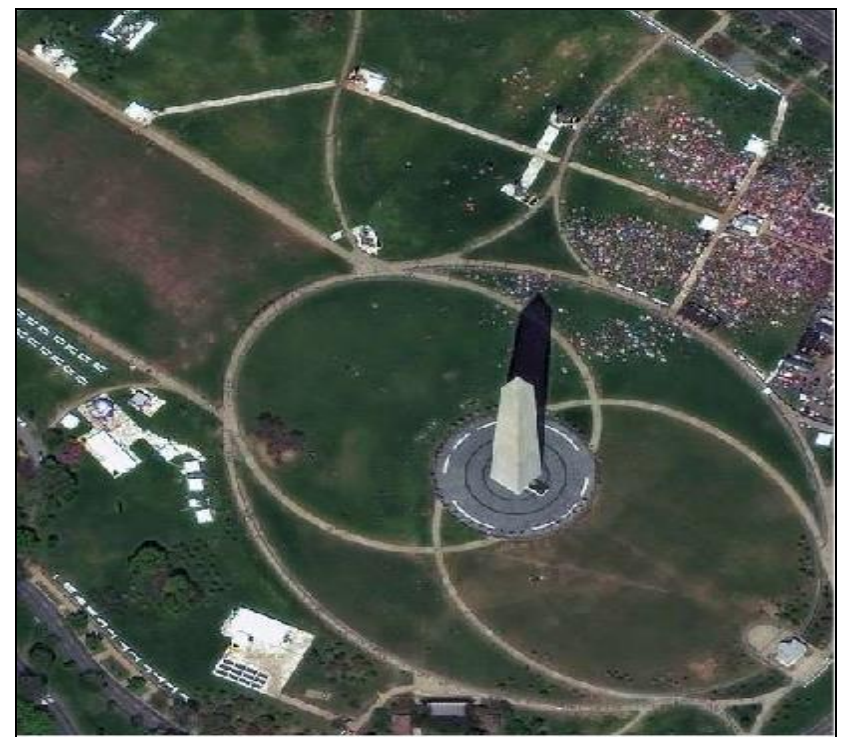

Figure 2. Original image

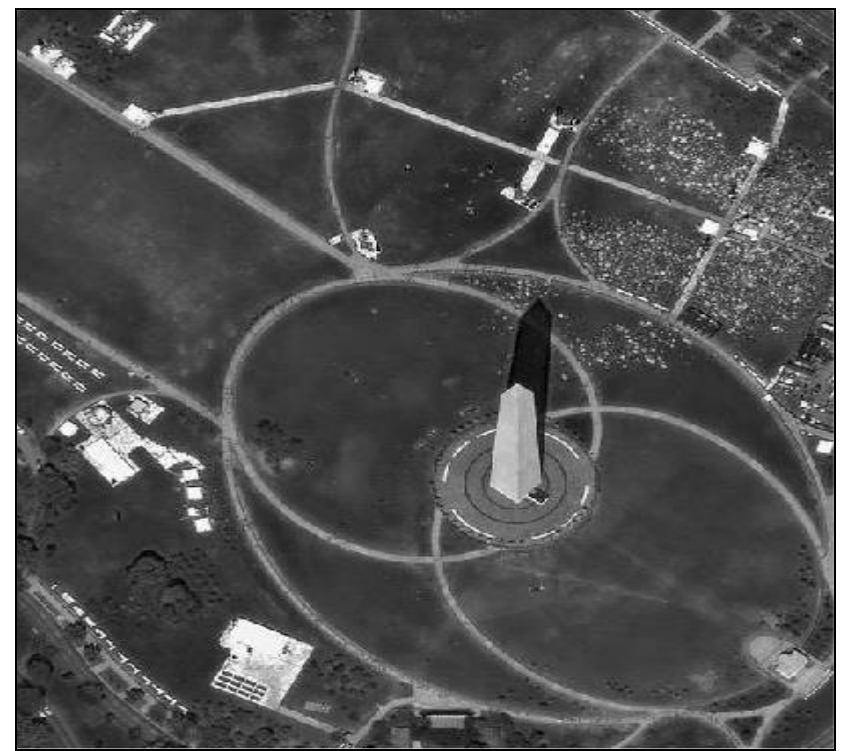

Figure 3. Image obtained after grayscale conversion

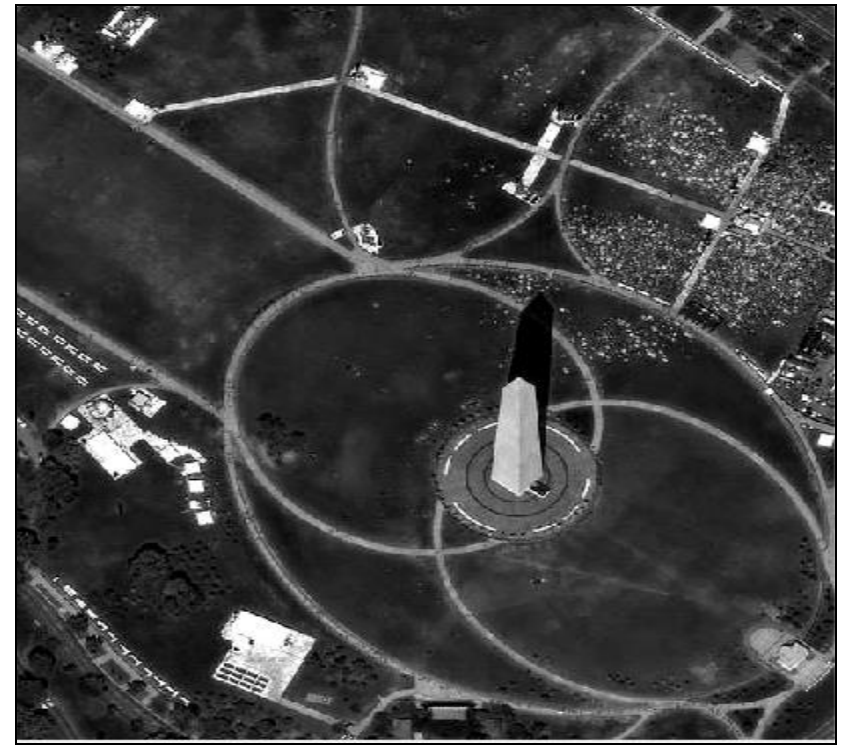

Figure 4. Image obtained after performing histogram equalization on grayscale image

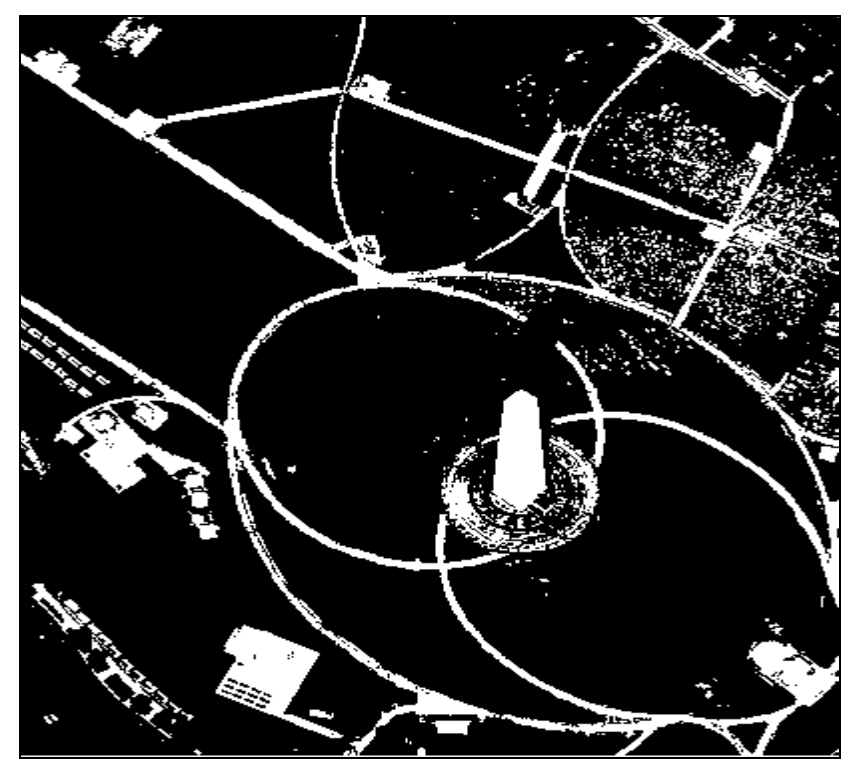

Figure 5. Image obtained after binarization

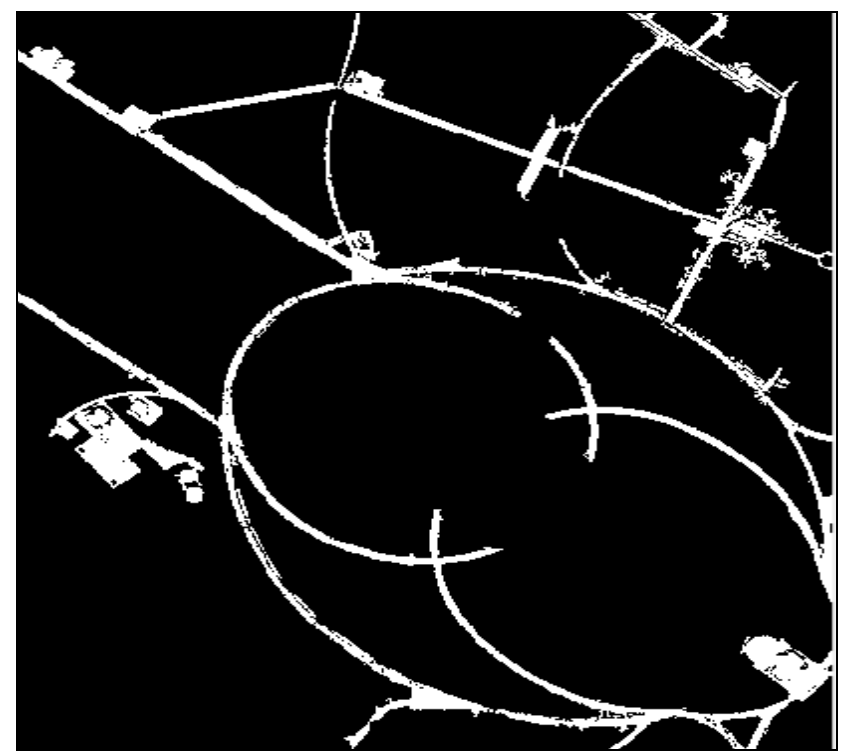

Figure 6. Image after connected component analysis

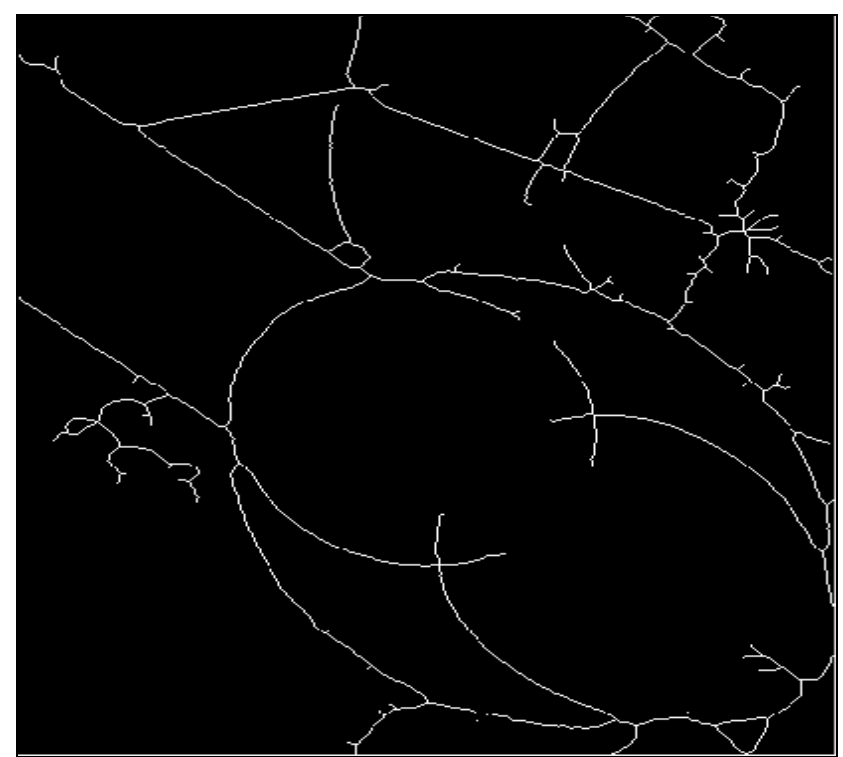

Figure 7. Image obtained after applying morphological 


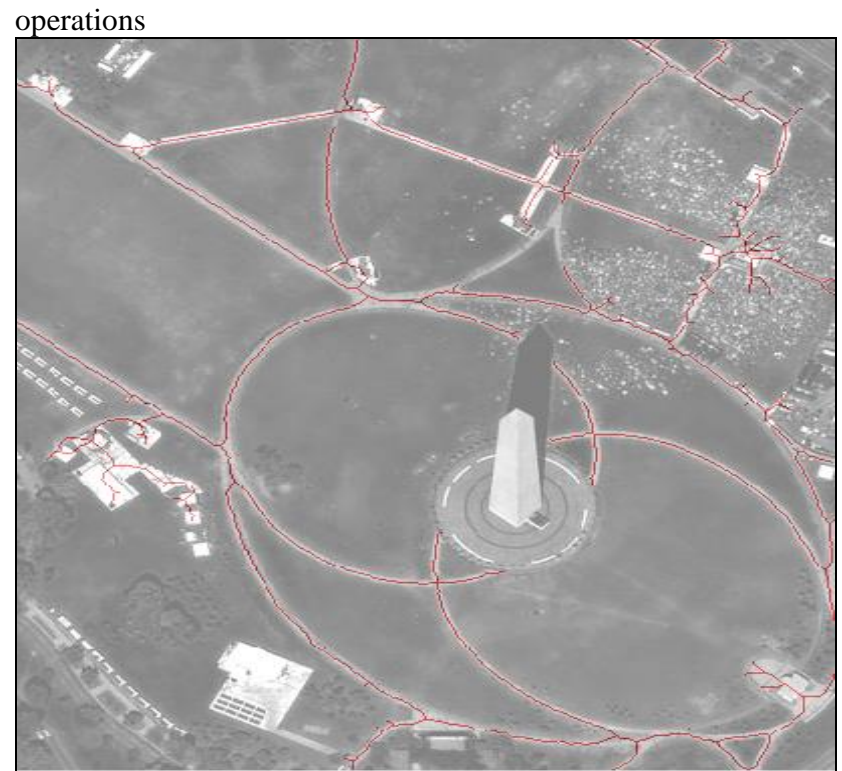

Figure 8. Detected road network

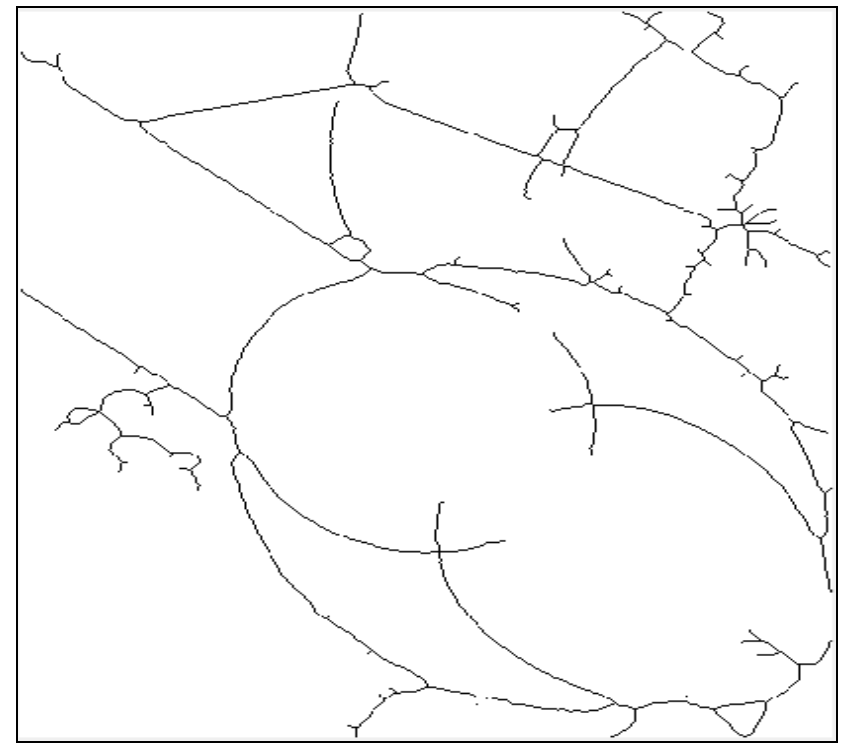

Figure 9. Extracted road network

\section{RESULTS AND DISCUSSION}

The results of proposed method of road identification and extraction are shown in above figures. The proposed method has been applied on high resolution satellite images. Figure 2 shows the original colored image. This image is of Washington DC captured by WorldView-3 on April 18, 2015. It is downloaded from DigitalGlobe website. Figure 3 shows the image obtained after the grayscale conversion of the original image. Figure 4 shows the image obtained after histogram equalization. In this difference between foreground objects and background has been enhanced. Figure 5 shows the binary conversion of image. In this, road areas have been converted to white pixels but there are some other objects also which have been converted to white pixels but they do not belong to road. Figure 6 shows image after connected component analysis. In this part, the objects which are not connected to road have been removed. Figure 7 shows the result of the morphological operations which are applied on the image. Small isolated pixels have been removed and road network has been reduced to thin lines. In Figure 8, identified road network is highlighted by red color on the grayscale image. This shows the centerline of the road. Figure 9 shows the extracted road network in which black pixels show the road network and other objects are shown by white pixels. After the extraction, as figure 9 shows, most of the road pixels have been obtained. Extra objects which are identified around the road area have been removed. Some areas that are connected to road network could not been removed. But most areas of road have been extracted.

The proposed method has been implemented to GUI so that any person can perform the road detection and extraction using this GUI. Operations such as grayscale conversion, connected component analysis etc can easily performed by using pushbuttons. User can browse the image to be processed. Image window has also been given in which image is shown after every operation. Two pushbutton 'Refresh' and 'Clear the image window' have also been added for removing the data of variable and for clearing the image window respectively. Four toggle buttons have been added for four morphological operations: area opening, closing, thinning and opening operation. For area opening, options for selecting the value of threshold have been added. The GUI in operation has been shown in figure 10 .

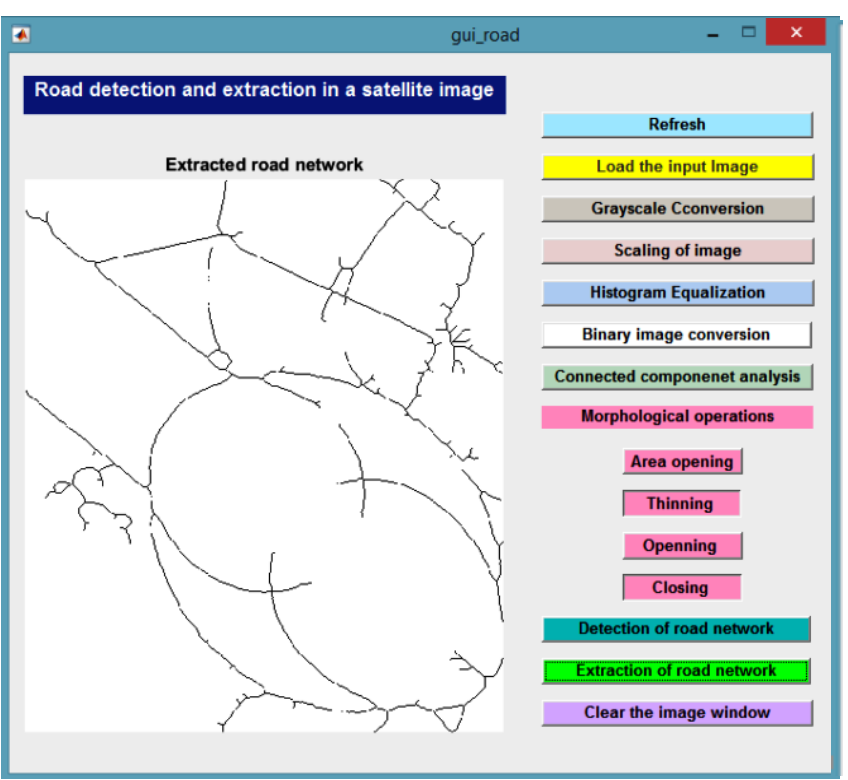

Figure 10. GUI developed in MATLAB

\section{CONCLUSIONS}

In this paper, road detection and extraction method has been proposed and implemented for high resolution satellite images. This method is based on Otsu's segmentation method, connected component analysis and morphological operations. In proposed method, high resolution satellite image is converted to grayscale image, rescaling of image was performed and then contrast of image was enhanced by histogram equalization. A threshold was selected by using Otsu's method and using this threshold, image was converted into binary image. After that, connected component analysis was performed which removed the areas which were not connected to road network. After that, morphological operations were used to refine the image. Then, extracted road network has been found. After road detection, a grayscale image with red colored road network was found. There are some limitations in this approach. The areas connected to road network could not been removed. To deal 
with this limitation, if width of objects could be calculated then this limitation could be removed by applying a threshold on width of objects.

\section{REFERENCES}

Alemu, M.M., 2016. Automated Farm Field Delineation and Crop Row Detection from Satellite Images. Geo-Information Science and Earth Observation of the University of Twente.

Alian, S., 2007. Identifying Curviness of Overpass Mountain Roads from Remote Sensing Data. International Institute For Geo-Information Science And Earth Observation.

Alvarez, J.M., Gevers, T., Lopez, A.M., 2009. Vision - Based Road Detection Using Road Models, in: IEEE International Conference on Image Processing. Cairo, pp. 2073-2076. https://doi.org/10.1109/ICIP.2009.54143211

Amo, M., Martínez, F., Torre, M., 2006. Road Extraction from Aerial Images Using A Region Competition Algorithm. IEEE Transactions on Image Processing 15, 1192-1201. https://doi.org/10.1109/TIP.2005.864232

Bao, J., Zhang, Y., Su, X., Zheng, R., 2018. Unpaved Road Detection Based on Spatial Fuzzy Clustering Algorithm. Journal on Image and Video Processing 26, 1-15. https://doi.org/10.1186/s13640-018-0260-3

Bin, L., Li, P., 2004. Object Extraction Based on Evolutionary Morphological Processing. Geo-spatial Information Science 7, 193-197. https://doi.org/10.1007/BF02826290

Chalekar, K., Yengantiwar, T., 2014. Review Paper on Image Contrast Enhancement Techniques. International Journal of Advanced Research in Computer Engineering \& Technology (IJARCET) 3, 909-911.

Chen, C., Qin, Q., Zhang, N., Li, J., Chen, L., Wang, J., Qin, X., Yang, X., 2014. Extraction of Bridges Over Water from High-Resolution Optical Remote-Sensing Images Based on Mathematical Morphology. International Journal of Remote Sensing 35, 3664-3682. https://doi.org/10.1080/01431161. 2014.915437

Chen, J., Pan, D., Mao, Z., 2009. Image-Object Detectable in Multiscale Analysis on High-Resolution Remotely Sensed Imagery. International Journal of Remote Sensing 30, 35853602. https://doi.org/10.1080/01431160802585348

Dal Poz, A.P., Zanin, R.B., do Vale, G.M., 2006. Automated Extraction of Road Network from Medium-and HighResolution Images. Pattern Recognition and Image Analysis 16, 239-248. https://doi.org/10.1134/S1054661806020118

Duta, N., 2000. Road Detection in Panchromatic SPOT Satellite Images, in: 15th International Conference on Pattern Recognition. Barcelona, pp. 308-311. https://doi.org/10.1109/ICPR.2009.902920

Dutra, L. V., 1999. Feature Extraction and Selection for ERS1/2 Insar Classification. International Journal of Remote Sensing 20, 993-1016. https://doi.org/10.1080/01431169 9213046
Dutra, L. V., Mascarenhas, N.D.A., 1984. Some Experiments With Spatial Feature Extraction Methods In Multispectral Classification. International Journal of Remote Sensing 5, 303313. https://doi.org/10.1080/01431168408948810

Foedisch, M., Takeuchi, A., 2004. Adaptive Road Detection Through Continuous Environment Learning, in: Internation Symposium on Information Theory. Washington DC, pp. 1621. https://doi.org/10.1109/AIPR.2004.9

Gambini, J., Mejail, M.E., Jacobo-Berlles, J., Frery, A.C., 2006. Feature Extraction in Speckled Imagery Using Dynamic Bspline deformable Contours Under the Model. International Journal of Remote Sensing 27, 5037-5059. https://doi.org/10.1080/01431160600702616

Gao, S., 2013. Research on Medical Image Processing Method Based on the Matlab, in: Informatics and Management Science V. London, pp. 687-694. https://doi.org/10.1007/978-1-44714796-1

Ge, Y., Bai, H., 2011. Multiple-Point Simulation-based Method for Extraction of Objects with Spatial Structure from Remotely Sensed Imagery. International Journal of Remote Sensing 32, 2311-2335. https://doi.org/10.1080/01431161003698278

Gioi, R.G. von, Jakubowicz, J., Morel, J.-M., Randall, G., 2008. On Straight Line Segment Detection. Journal of Mathematical Imaging and Vision 32, 313-347. https://doi.org/10.1007/s10851-008-0102-5

Gonzalez, R.C.., Woods, R.E., 2008. Digital Image Processing, Nueva Jersey. https://doi.org/10.1049/ep.1978.0474

Gruen, A., Li, H., 1997. Semi-Automatic Linear Feature Extraction by Dynamic Programming and LSB-Snakes. Photogrammetric Engineering and Remote Sensing 63, 985995.

Haigang, S., Deren, L., Jianya, G., 2003. Automatic Change Detection for Road Networks from Images Based on GIS. Geospatial Information Science 6, 44-50. https://doi.org/10.1007/BF02826949

Hassaballah, M., Abdelmgeid, A.A., Alshazly, H.A., 2016. Image Feature Detectors, Descriptors and Matching, in: Awad, A.I., Hassaballah, M. (Eds.), Image Feature Detectors and Descriptors. Springer International Publisher, pp. 11-45. https://doi.org/10.1007/978-3-319-28854-3

He, Y., Wang, H., Zhang, B., 2004. Color-Based Road Detection in Urban Traffic Scenes. IEEE Transaction on Intelligent Transportation Systems 5, 309-318. https://doi.org/10.1109/TITS.2004.838221

Hemanth D, J., Jude, A., 2012. Image Pre-processing and Feature Extraction Techniques for Magnetic Resonance Brain Image Analysis, in: Computer Applications for Communication, Networking and Digital Contents. pp. 349-356. https://doi.org/10.1007/978-3-642-35594-3

Hu, J., Razdan, A., Femiani, J.C., Cui, M., Wonka, P., 2007. Road Network Extraction and Intersection Detection from Aerial Images by Tracking Road Footprints. IEEE Transactions on Geoscience and Remote Sensing 45, 4144-4157. https://doi.org/10.1109/TGRS.2007.906107 
Hu, X., Zhang, Z., Li, J., 2009. Linear Feature Extraction Using Adaptive Least - Squares Template Matching and a Scalable Slope Edge Model. International Journal of Remote Sensing 30, 3393-3407. https://doi.org/10.1080/01431160802562198

Huang, Z., Jia, X., 2012. Integrating Remotely Sensed Data, GIS and Expert Knowledge To Update Object-Based Land use/land Cover Information. International Journal of Remote Sensing 33, 905-921. https://doi.org/10.1080/01431161.2010 .536182

Jeon, B.-K., Jang, J.-H., Hong, K.-S., 2002. Road Detection in Spaceborne SAR Images using a Genetic Algorithm. IEEE Transactions on Geosience and Remote Sensing 40, 22-29. https://doi.org/10.1109/36.981346

Kapoor, D., Kumar, M., Pant, P., 2014. Automated Object Recognition From High Resolution Satellite Images : A Review. International Journal of Advanced Research in Computer Engineering \& Technology (IJARCET) 3, 4277-4282.

Kaur, S., Ritika, 2013. Contrast Enhancement Techniques for Images - A Visual Analysis. International Journal of Computer Applications 64, 20-25.

Kawashima, T., Shirakawa, Y., Aoki, Y., 1994. Multisensorbased object identification using uncertain geometric models. Advanced Robotics 8, 31-43. https://doi.org/10.1163/156855 $394 X 00031$

Keaton, T., Brokish, J., 2002. A Level Set Method for the Extraction of Roads from Multispectral Imagery, in: Applied Imagery Pattern Recognition Workshop. Washington DC, pp. 141-147. https://doi.org/10.1109/AIPR.2002.1182268

Lak, A.M., Zoej, M.J.V., Mokhtarzade, M., 2016. A New Method for Road Detection in Urban Areas using HighResolution Satellite Images and Lidar Data Based on Fuzzy Nearest-Neighbor Classification and Optimal Features. Arabian $\begin{array}{llll}\text { Journal of } & \text { Geosciences }\end{array}$ https://doi.org/10.1007/s12517-016-2374-1

Lal, S., Kumar, R., Chandra, M., 2013. An Improved Method for Contrast Enhancement of Real World Hyperspectral Images, in: International Conference on Heterogenous Networking For Quality, Reliability, Security and Robustness. pp. 273-282. https://doi.org/10.1007/978-3-642-37949-3_23

Leelarani, B., Rameshnaidu, B., 2015. Recognition of License Plate Numbers Using Image Processing Technique and Genetic Algorithm. International Journal of Engineering Development and Research 3, 1-5.

Li, Y., Briggs, R., 2009. Automatic Extraction of Roads from High Resolution Aerial and Satellite Images with Heavy Noise, in: World Academy of Science, Engineering and Technology. pp. 416-422.

Lin, X., Liu, Z., Zhang, J., Shen, J., 2009. Combining Multiple Algorithms for Road Network Tracking from Multiple Source Remotely Sensed Imagery: A Practical System and Performance Evaluation. Sensors 9, 1237-1258. https://doi.org/10.3390/ s90201237

Maboudi, M., Amini, J., Hahn, M., Saati, M., 2017. ObjectBased Road Extraction from Satellite Images using Ant Colony
Otimization. International Journal of Remote Sensing 38, 179198. https://doi.org/10.1080/01431161.2016.1264026

Malladi, C., 2017. Detection of Objects in Satellite images using Supervised and Unsupervised Learning Methods. Blekinge Institute of Technology.

Mena, J.B., 2003. State of the Art on Automatic Road Extraction for GIS update: A Novel Classification. Pattern Recognition Letters 24, 3037-3058. https://doi.org/10.1016/ S0167-8655(03)00164-8

Mnih, V., Hinton, G.E., 2010. Learning to Detect Roads in High-Resolution Aerial Images, in: European Conference on Computer Vision. Greece, pp. 210-223. https://doi.org/10.10 07/978-3-642-15567-3_16

Mohammadzadeh, A., ValadanZoej, M.J., Tavakoli, A., 2007. Automatic Class Mean Calculation of Road Surface From Ikonos Images Using Fuzzy Logic and Particle Swarm Optimization, in: Photogrammetric Image Analysis. ISPRS, Munich, pp. 119-124.

Mohammadzadeh, A., Zoej, M.J.V., Tavakoli, A., 2009. Automatic Main Road Extraction from High Resolution Satellite Imageries by Means of Particle Swarm Optimization Applied to a Fuzzy-Based Mean Calculation Approach. Journal of the Indian Society of Remote Sensing 37, 173-184. https://doi.org/10.1007/s12524-009-0021-y

Parrinello, T., Vaughan, R.A., 2002. Multifractal Analysis and Feature Extraction in Satellite Imagery. International Journal of Remote Sensing 23, 1799-1825. https://doi.org/10.1080/014 31160110075820

Parvathi, K., Rao, B.S.P., Rao, T.V., Reddy, K.M., 2010. Feature Extraction from Satellite Images of Hilly Terrains using Wavelets and Watersheds. International Journal of Remote Sensing 31, 5855-5866. https://doi.org/10.1080/01431161.201 0.512309

Peng, S., Guodong, Y., Xuefeng, N., Xuqing, Z., Fulei, Z., Tianqi, T., 2013. Information Extraction of High-Resolution Remotely Sensed Image Based on Multiresolution Segmentation, in: ISPRS /IGU/ICA Joint Workshop on Borderlands Modelling and Understanding for Global Sustainability. ISPRS, Beijing, pp. 117-121. https://doi.org/10.5194/isprsarchives-XL-4-W3-117-2013

Qu, Z., Zhang, L., 2010. Research on Image Segmentation Based on the Improved Otsu Algorithm, in: $2^{\text {nd }}$ International Conference on Intelligent Human-Machine Systems and Cybernetics. Jiangsu, pp. 228-231. https://doi.org/10.1109/IH MSC.2010.157

Reddy, D.S., Padmaja, M., 2016. Extraction of Roads from High Resolution Satellite Images by Means of Adaptive Global Thresholding \& Morphological Operations. International Journal of Scientific \& Engineering Research, 7, 559-563.

Rianto, Y., Kondo, S., Kim, T., 1999. Detection of Roads from Satellite Image Using the Optimal Search, in: International Conference on Image Analysis and Processing. Venice, pp. 804-809. https://doi.org/10.1142/S0218001400000635 
Schowengerdt, R.A., 2007. Remote Sensing: Models and Methods for Image Processing, 3rd ed. Elsevier.

Singh, K.K., Pal, K., Nigam, M.J., 2012. Shadow Detection and Removal from Remote Sensing Images using NDI and Morphological Operators. International Journal of Computer Applications 42, 37-40. https://doi.org/10.5120/5731-7805

Singh, P.P., Garg, R.D., 2014. A Two-Stage Framework for Road Extraction from High-Resolution Satellite Images by using Prominent Features of Impervious Surfaces. International Journal of Remote Sensing 35, 8074-8107. https://doi.org/10. 1080/01431161.2014.978956

Singh, P.P., Garg, R.D., 2013. Automatic Road Extraction from High Resolution Satellite Image using Adaptive Global Thresholding and Morphological Operations. Journal of Indian Socociety Remote Sensing 41, 631-640. https://doi.org/10. 1007/s12524-012-0241-4

Skulimowski, A.M.J., 2012. Intelligent Science and Intelligent Data Engineering, Lecture Notes in Computer Science (including subseries Lecture Notes in Artificial Intelligence and Lecture Notes in Bioinformatics). https://doi.org/10.1007/9783-642-31919-8

Soni, A., Pandey, N., Halarnkar, P., 2014. Review on Image Object Extraction. International Journal of Current Engineering and Technology 4, 864-869.

Sujatha, C., Selvathi, D., 2015. Connected Component-Based Technique for Automatic Extraction of Road Centerline in High Resolution Satellite Images. Eurasip Journal on Image and Video Processing 8, 1-16. https://doi.org/10.1186/s13640-0150062-9

Sundaram, M., Ramar, K., Arumugam, N., Prabin, G., 2011. Histogram Modified Local Contrast Enhancement for Mammogram Images. Applied Soft Computing Journal 11, 5809-5816. https://doi.org/10.1016/j.asoc.2011.05.003

Tiwari, D., Saroha, G.P., Bhushan, U., 2012. Road Network Extraction from Satellite Images By Active Contour (Snake) Model And Fuzzy C-Means. International Journal of Advanced Research in Computer Science and Software Engineering 2, 195-198.

Valero, S., Chanussot, J., Benediktsson, J.A., Talbot, H., Waske, B., 2010. Advanced Directional Mathematical Morphology for the Detection of the Road Network in Very High Resolution Remote Sensing Images. Pattern Recognition Letters 31, 1120-1127. https://doi.org/10.1016/j.patrec.2009. 12.018

Veda, D., Suma, B., 2016. Automatic Road Extraction from Satellite Image by Difference of Gaussian and Convolution Overlap Add Method. International Journal of Advanced Networking \& Applications 239-243.

Vijayan, S.C., R.L., J., Anilkumar, 2016. Histogram Based Connected Component Analysis for Character Segmentation. International Journal of Scientific and Research Publications 6, 200-202.

Wang, J., Qian, J., Ma, R., 2013. Urban Road Information Extraction from High Resolution Remotely Sensed Image based on Semantic Model, in: International Conference on Geoinformatics. IEEE, Kaifeng, pp. 1-5. https://doi.org/10.1109/Geoinformatics.2013.6626045

Wang, J., Qin, Q., Gao, Z., Zhao, J., Ye, X., 2016. A New Approach to Urban Road Extraction Using High-Resolution Aerial Image. ISPRS International Journal of Geo-Information 5, 1-12. https://doi.org/10.3390/ijgi5070114

Wang, M., Zhang, S., 2011. Road Extraction from HighSpatial-Resolution Remotely Sensed Imagery by Combining Multi-Profile Analysis and Extended Snakes Model. International Journal of Remote Sensing 32, 6349-6365. https://doi.org/10.1080/01431161.2010.508801

Wang, S., Liu, C., Wu, S., Qianqian, N., Wang, Y., Shia, Z., Haifeng, Z., 2012. Automatic Extraction of Foreground Objects from Mars Images. Geo-spatial Information Science 15, 17-25. https://doi.org/10.1080/10095020.2012.708147

Wu, W., ShuFeng, G., 2009. Research on Unstructured Road Detection Algorithm Based on the Machine Vision, in: AsiaPacific Conference on Information Processing. IEEE, Shenzhen, pp. 112-115. https://doi.org/10.1109/APCIP.2009 .164

Zeng, G., 2008. Real-time Automatic Linear Feature Detection In Images. Electrical and Computer Engineering Commons Recommended. Clemson University.

Zhang, H., Lin, H., Li, Y., Zhang, Y., 2013. Feature Extraction for High-Resolution Imagery Based on Human Visual Perception. International Journal of Remote Sensing 34, 11461163. https://doi.org/10.1080/01431161.2012.718459 